\title{
Thorough analysis of input physics in CESAM and CLÉS codes
}

\author{
Josefina Montalbán • Yveline Lebreton . \\ Andrea Miglio • Richard Scuflaire • Pierre Morel • \\ Arlette Noels
}

Received: 5 December 2007 / Accepted: 7 April 2008 / Published online: 23 April 2008

(C) Springer Science+Business Media B.V. 2008

\begin{abstract}
This contribution is not about the quality of the agreement between stellar models computed by CESAM and CLÉS codes, but more interesting, on what ESTA-Task 1 run has taught us about these codes and about the input physics they use. We also quantify the effects of different implementations of the same physics on the seismic properties of the stellar models, that in fact is the main aim of ESTA experiments.
\end{abstract}

Keywords Stars: internal structure - Stars: oscillations . Stars: numerical models

PACS 97.10.Cv $\cdot$ 97.10.Sj $\cdot 95.75 . \mathrm{Pq}$

\section{Introduction}

The goal of ESTA-Task 1 experiment is to check the evolution codes and, if necessary, to improve them. The results of

J. Montalbán $(\varangle) \cdot$ A. Miglio · R. Scuflaire · A. Noels Institut d'Astrophysique et Geophysique, Université de Liège, allée du 6 Août 17, 4000 Liège, Belgium

e-mail: J.Montalban@ulg.ac.be

A. Miglio

e-mail: A.Miglio@ulg.ac.be

R. Scuflaire

e-mail: R.Scuflaire@ulg.ac.be

Y. Lebreton

Observatoire de Paris, GEPI, CNRS UMR 8111, 5 Place Janssen,

92195 Meudon, France

e-mail: Yveline.Lebreton@obspm.fr

P. Morel

Département Cassiopée, CNRS UMR 6202,

Observatoire de la Côte d'Azur, Nice, France

e-mail: Pierre.Morel@obs-nice.fr
Task 1 comparisons were presented in Monteiro et al. (2006) and Lebreton et al. (2008a) for a set of stellar models representative of potential CoRoT targets. The models calculated for TASK 1 were based on rather simple input physics. Moreover, a great effort was done to reduce at maximum the differences between computations by fixing the values of fundamental constants and the physics to be used (see Lebreton et al. 2008b). In spite of that, some differences among stellar models computed by different codes persist.

In CESAM and CLÉS computations we paid attention to adopt, not only the same fundamental constants and metal mixture (Grevesse and Noels 1993, thereafter GN93), but also the same isotopic ratios and atomic mass values. Nevertheless, even if the same metal mixture, opacity tables and equation of state were adopted, there is still some freedom on their implementation. In this paper we analyze these different implementations and estimate the consequent effects on the stellar structure and on the seismic properties of the theoretical models. In Sect. 2 we study the equation of state and in Sect. 3 the differences in the opacity tables. The nuclear reaction rates are discussed in Sect. 4 and the effect of different surface boundary conditions in Sect. 5. Finally, in Sect. 6 we analyze the differences due to different numerical techniques.

\section{Equation of State}

As fixed in ESTA we used the OPAL2001 (Rogers and Nayfonov 2002) equation of state which is provided in a tabular form. In CESAM the quantities: density, $\rho$, internal energy, $E$, the compressibilities $\chi_{T}=(\partial \ln P / \partial \ln T)_{\rho}$ and $\chi_{\rho}=(\partial \ln P / \partial \ln \rho)_{\mathrm{T}}$, the adiabatic indices $\Gamma_{1}, \Gamma_{2} /\left(\Gamma_{2}-1\right)$, $\Gamma_{3}-1$, and the specific heat at constant volume $C_{V}$, are obtained from the variables $P, T, X$ and $Z$ (respectively pres- 


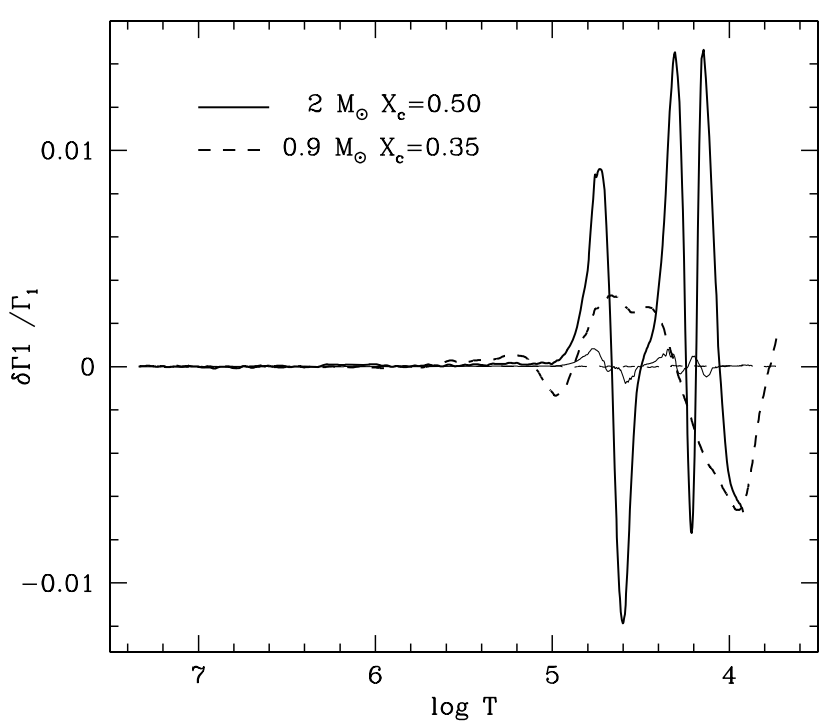

Fig. 1 Relative differences in the $\Gamma_{1}$ values provided for a given $(\rho, T)$ structure by CESAM and CLÉS EOS routines. The thick lines correspond to $\Gamma_{1}$ values derived from $C_{V}$ tabulated values while the thin ones are derived from the tabulated $\Gamma_{1}$. Solid lines corresponds to a $2 M_{\odot}$ model with a central hydrogen mass fraction $X_{\mathrm{c}}=0.50$, and dashed ones to a $0.9 M_{\odot}$ star in the middle of the main-sequence

sure, temperature, hydrogen and heavy element mass fraction) using the interpolation package provided on the OPAL web site, and the specific heat at constant pressure $\left(C_{p}\right)$ is derived from $\left(\Gamma_{3}-1\right)$. On the other hand, CLÉS interpolates only $C_{V}, P, \chi_{\rho}$ and $\chi_{T}$ in the OPAL EOS tables by a method ensuring the continuity of first derivatives at cell boundaries in the four-dimensional space defined by the variables $\rho, T$, $X$ and $Z$. The other thermodynamic quantities $\Gamma_{1},\left(\Gamma_{3}-1\right)$ and $C_{p}$ are derived from the values of $C_{V}, P, \chi_{\rho}$ and $\chi_{T}$ by means of the thermodynamic relations.

As a first step we want to disentangle the differences in the thermodynamic quantities from their effects on the stellar structure. We estimate therefore the intrinsic differences between the equation of state used in CESAM and in CLÉS. To this purpose we computed the differences between the thermodynamic quantities from the corresponding EoS routines, for a stellar structure defined by $\rho, T, X$ and $Z$ values. In Fig. 1 (thick lines) we show the result of $\Gamma_{1}$ comparison for two different stellar models, a $2 M_{\odot}$ model with a mass fraction of hydrogen in the center $X_{\mathrm{c}}=0.50$ (solid line) and a $0.9 M_{\odot}$ model with $X_{\mathrm{c}}=0.35$ (dashed line). By comparing also the other thermodynamic quantities we found that the largest discrepancies between CESAM and CLÉS EoS occurs for $\log T<5$ (corresponding to the partial $\mathrm{He}$ and $\mathrm{H}$ ionization regions), and they are, at maximum, of the order of $2 \%$ for $\Gamma_{1}$, and of $5 \%$ for $\nabla_{\text {ad }}$ and $C_{p}$. By using the OPAL interpolation routine in CLÉS, we verified that the different interpolation schemes used in CESAM and CLÉS can only account for an uncertainty of $0.05 \%$ in $P, 0.2 \%$ in $\Gamma_{1}$, and $0.5 \%$ in $\nabla_{\mathrm{ad}}$ and $C_{p}$. These remaining differences are probably explained by the fact that CESAM uses as variables $(P, T)$ and uses subroutine rhoofp of OPALpackage to transform $(P, T)$ into $(\rho, T)$, while CLÉS uses directly $(\rho, T)$. Nevertheless, those discrepancies are an order of magnitude smaller than the differences between CESAM and CLÉS EoS.

As it was already noted by Boothroyd and Sackmann (2003), some inconsistencies existed between thermodynamic quantities tabulated in OPAL EOS: "for the OPAL EOS (Rogers et al. 1996), we found that there were significant inconsistencies when we compared their tabulated values of $\Gamma_{1}, \Gamma_{2} /\left(\Gamma_{2}-1\right)$, and $\left(\Gamma_{3}-1\right)$ to the values calculated from their tabulated values of $P, C_{V}, \chi_{\rho}$, and $\chi_{T}$. ... Preliminary tests indicate that this OPAL2001 EOS has larger but smoother inconsistencies in its tabulated thermodynamic quantities...”. As a consequence of these inconsistencies, the choice of the basic thermodynamic quantities is not irrelevant, and it was shown by Roxburgh (2005, private communication), that the choice done in CLÉS was the worst one. A direct comparison with the values of $C_{V}$ computed from the derivative of the internal energy as tabulated in OPAL EOS, showed that the OPAL tabulated $C_{V}$ was affected by a large inaccuracy.

The OPAL team acknowledged afterwards the $C_{V}$-issue and recommended not to use it. The EoS tables used in CLÉS have then been changed by replacing the tabulated $C_{V}$ value by that obtained from the tabulated values of $P, \chi_{\rho}, \chi_{T}$, and $\Gamma_{1}$. The remaining discrepancies $(\sim 0.2 \%)$ between the CESAM $\Gamma_{1}$ values and those from the new CLÉS-EoS table (hereafter called CLÉS-EoS- $\Gamma_{1}$ to tell it apart from the original one CLÉS-EoS- $C_{V}$ ) are due to the different interpolation routine. As shown in Fig. 1 (thin lines) the discrepancies are much smaller for a solar like than for a $2 M_{\odot}$ model and they appear mainly in the ionization regions.

Concerning the quantities that in CLÉS are obtained from thermodynamic relations and in CESAM from interpolation in OPAL tables, the differences come in part from the interpolation routine and in part from the remaining, even if much smaller, inconsistencies between the tabulated values of $\Gamma_{1}, \Gamma_{2} /\left(\Gamma_{2}-1\right)=\nabla_{\mathrm{ad}}^{-1}$, and $\left(\Gamma_{3}-1\right)$. For instance, the values of $C_{V}$ derived from $\Gamma_{1}$ may differ by $0.5 \%$ from the corresponding value obtained from $\left(\Gamma_{3}-1\right)$, and that occurs always in the $\mathrm{H}$ and $\mathrm{He}$ ionization regions. The problem is that even if the thermodynamic relations to derive the adiabatic indices seem more physical, there is some numerical incoherence. In fact, the derivatives of interpolated (very often polynomial) quantities do not fit in those of the interpolated functions (whose behavior is far from polynomial one).

All the CLÉS models involved in Task 1 and Task 3 comparisons (Lebreton et al. 2008a) were recomputed with CLÉS-EoS- $\Gamma_{1}$, but the models used for comparisons presented in Monteiro et al. (2006) were not. In fact, most of 


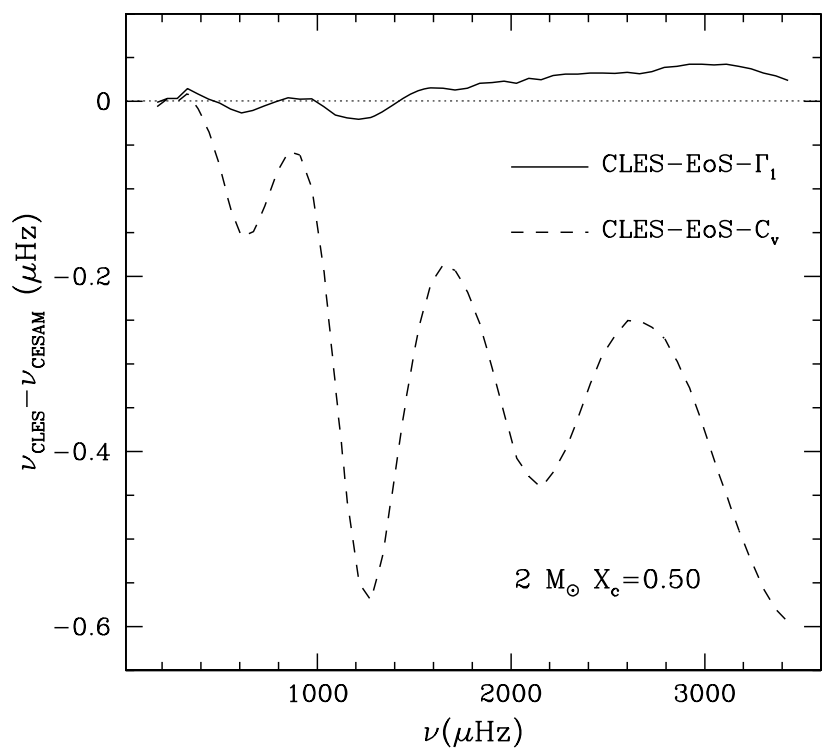

Fig. 2 Frequency differences between CESAM and CLÉS $2 M_{\odot}$ models. The two different curves correspond to CLES models computed by using the two different EoS tables (see text)

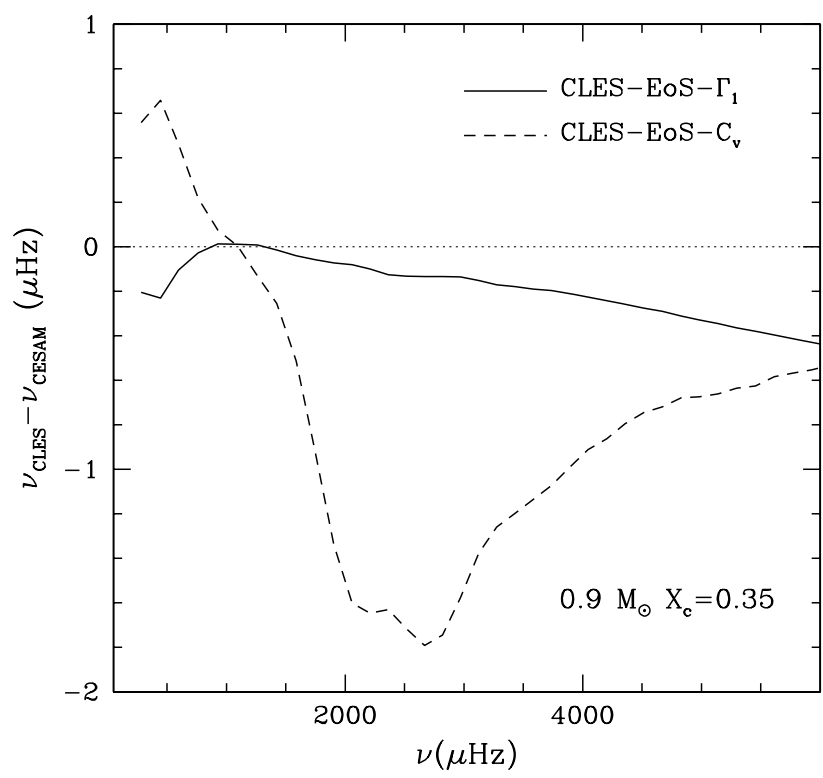

Fig. 3 As Fig. 2 for $0.9 M_{\odot}$ model

the frequency differences found in that paper came from CLÉS-EoS- $C_{V}$. The effect of EoS differences on the seismic properties are illustrated in Fig. 2 for a $2 M_{\odot}$ model and in Fig. 3 for the solar like model. In those figures we plot the frequency differences of $\ell=0$ modes for CESAM models and two types of CLÉS ones: those computed with EoS- $C_{V}$ (dashed lines) and those computed with EoS- $\Gamma_{1}$ (solid line). The period of the oscillatory signature shown by $\Delta v\left(v_{\text {CLES }}-v_{\text {CESAM }}\right)$ in Fig. 2 is related to the acoustic depth where models differ. A Fourier transform of $\Delta v$ shows clearly that the oscillation is linked to the $\Gamma_{1}$ differences. Moreover, the comparison of Fig. 3 with Fig. 6 in Monteiro et al. (2006) confirms that also for the Case1.1 model (see e.g. Lebreton et al. 2008a), the maximum difference of almost $2 \mu \mathrm{Hz}$ between CESAM and CLÉS models found by Monteiro et al. (2006) was due to the inconsistency between the tabulated $C_{V}$ and adiabatic indices.

\section{Opacities}

ESTA specifications require the use of OPAL96 opacity tables (Iglesias and Rogers 1996) complemented at low temperatures by the Alexander and Ferguson (1994) (thereafter AF94) tables. CESAM uses OPAL tables provided by C. Iglesias, prior to their availability on the web site, and interpolates in the opacity tables by means of a fourpoint Lagrangian interpolation. The OPAL opacity tables used by CLÉS were picked up later on the OPAL web site and smoothed according to the prescription found there (xztrin21.f routine), we will call them thereafter OPAL96-S. Furthermore, the interpolation method in CLÉS opacity routine is the same as that used in EoS table interpolation. In both codes the metal mixture adopted in the opacity tables is the GN93 one.

To disentangle the differences in the opacity computations from the differences in the stellar structure, we proceed as in EoS table analysis, that is, we estimate the intrinsic differences in the opacity $(\kappa)$ by comparing the $\kappa$ values provided by CESAM and by CLÉS routines for the same stellar structure. The results of these comparisons are shown in Figs. 4 and 5, where we plot for two different stellar models the opacity relative differences $\left(\kappa_{\mathrm{CESAM}}-\kappa_{\mathrm{CLES}}\right) / \kappa$ as a function of the local temperature and of the relative radius. From comparisons of different models it results that the opacity discrepancies depend on the mass of the stellar model and, for a given mass, on the evolutionary state as well. Moreover, a peaked feature at $\log T \simeq 4$ which can reach values of the order of $5 \%$, appears in all the comparisons. This is a consequence of the differences between OPAL and AF94 opacities in the domain [9000 K-12000 K] and of the different method used in CLÉS and CESAM to assemble AF94 and OPAL tables. CLÉS uses the procedure described in Scuflaire et al. (2008b) that ensures a smooth passage between both tables, while CESAM searches for the point of minimum discrepancy between OPAL and AF94. In the interior regions the differences between CESAM and CLÉS opacities do not present the oscillatory behavior that we would expect if these differences resulted from the interpolation schemes. On the contrary, the CESAM opacities are systematically larger (by 1-2\%) than the CLÉS ones in the region $\log T \in[5.5,7]$ of $2 M_{\odot}$ model.

Even if the metal mixture to be used in opacity computations is fixed (GN93), there may be some uncertainties in 


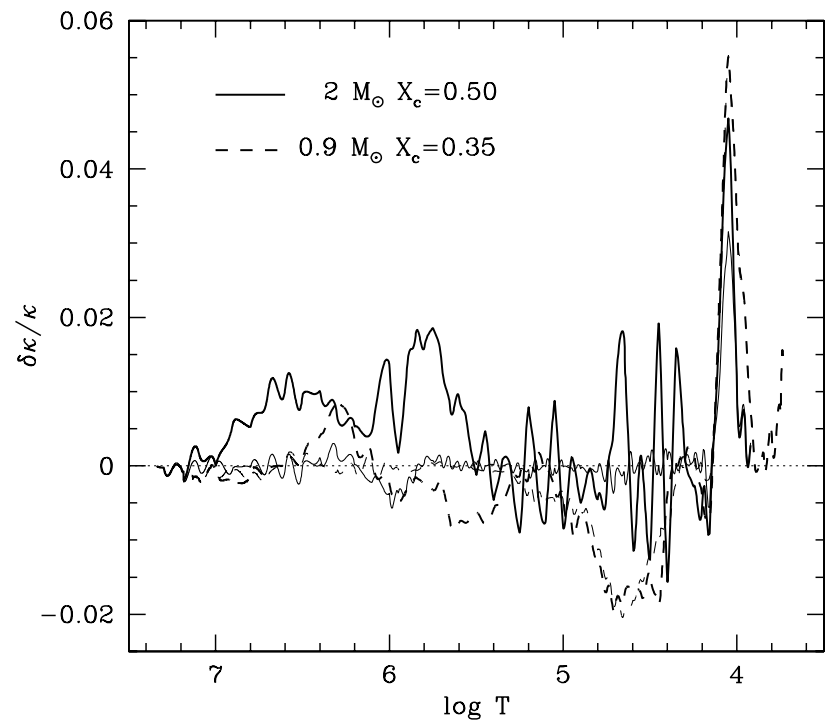

Fig. 4 Relative differences in the opacity values provided for a given $(\rho, T)$ structure by CESAM and CLÉS opacity routines. The thick lines correspond to the $\kappa$ values derived from the smoothed OPAL tables, while the thin ones were obtained by including in the CLES opacity routine the OPAL tables without smoothing. Solid lines corresponds to a $2 M_{\odot}$ model with $X_{\mathrm{c}}=0.50$, and dashed ones to a $0.9 M_{\odot}$ star in the middle of the main-sequence

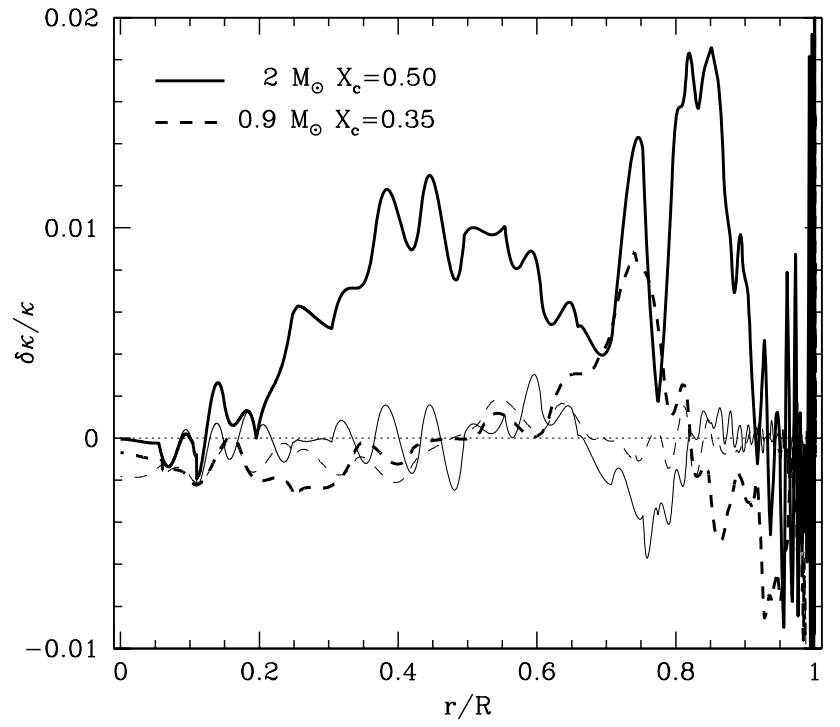

Fig. 5 The same differences as in Fig. 4 but plotted as a function of the relative stellar radius

its definition. For instance, OPAL uses atomic masses that do not correspond to the values given by the isotopic ratios in Anders and Grevesse (1989). In particular there is a difference of $0.5 \%$ for Neon, and $10 \%$ for Argon. Moreover, OPAL opacity tables are computed for 19 elements, while the GN93 mixture contains 23 elements. There are two options: either to ignore the mass fraction of $\mathrm{F}, \mathrm{Sc}, \mathrm{V}$, and $\mathrm{Co}$, or to allot the abundances of these elements among the close neighbors. We have analyzed the effects of these uncertainties on the opacity values, but they turned out to be of the same order of the accuracy in OPAL data $(0.1-0.2 \%)$. Hence, they cannot account for the discrepancy between CESAM and CLÉS opacities.

The other important difference between CESAM and CLÉS opacities is on whether they use the OPAL smoothing routine or not. In fact, the OPAL opacity tables are affected by somewhat random numerical errors of a few percent. To overcome undesirable effects the OPAL web site suggests to pass the original tabular data through a smoothing filter before interpolating for $Z, X, \log T$, and $R$ (with $R=\rho / T_{6}^{3}$ ). A direct comparison between the original and smoothed opacity values have shown a difference larger than $2 \%$ for $\log R=-4$ and -3.5 and $\log T \in[5.5,7]$. These differences decrease for larger and smaller values of $\log R$.

We have computed new opacity tables for the CLÉS opacity routine without passing through the smoothing filter (CLÉS-OPAL96 instead of CLÉS-OPAL96-S). The comparison between CESAM and new CLÉS opacity computations are also shown in Figs. 4, 5 (thin lines). We note that when both codes use similar OPAL96 tables, the discrepancies in the internal regions almost disappear. The remaining differences are due to the interpolation schemes and to the small differences in GN93 definition. The feature at $\log T \sim 4$ is still there since the method used in CLÉS to assemble AF94 and OPAL96 tables is the same as in CLÉS-OPAL96-S.

At variance with the EOS tables, where an error was detected and acknowledged by the OPAL team, we do not have any argument to prefer the smoothed to the original opacity tables, and we think that the differences between both groups of results must be considered an estimate of the precision of current stellar modeling. Therefore, the Liége group decided to provide for Task 1 and Task 3 comparisons (Lebreton et al. 2008a) the modes computed with the standard tables in CLÉS, that is OPAL96-S. A part of the differences between CESAM and CLÉS models that were reported in Lebreton et al. (2008a) should be hence due to the opacity tables we used. In order to estimate these effects we have recomputed with CLÉS and OPAL96 tables (without smoothing) the models for all the cases in TASK 1 (see Table 1 in Lebreton et al. 2008a). In the next three sections we present the effect of the opacity uncertainty on: the global parameters, the stellar structure, and on the seismic properties.

\subsection{Effects on global stellar parameters}

In general, the change of opacity tables decreases the discrepancies between the stellar global parameters provided by CLÉS and CESAM. In Table 1 we collect the differences (in percent) in radius, luminosity, central density, and central temperature between pairs of models for the Task 1 targets. Columns labeled A give the differences 
Table 1 Global parameter differences, in percent, between Task 1target models computed by CLÉS with OPAL opacity tables in the two versions: smoothed (OPAL96-S) and not smoothed (OPAL96). Stellar radius $(\Delta R)$, luminosity $(\Delta L)$, central density $\left(\Delta \rho_{c}\right)$ and central temperature $\left(\Delta T_{c}\right)$. Columns labeled $\mathrm{A}$ give the differences $X_{\text {CLES-OPAL96-S }}-X_{\text {CLES-OPAL96, }}$ and columns B and C the differences with CESAM, that is, $X_{\text {CLES-OPAL96-S }}-X_{\text {CESAM }}$ and $X_{\text {CLES-OPAL96 }}-X_{\text {CESAM }}$ respectively

\begin{tabular}{|c|c|c|c|c|c|c|c|c|c|c|c|c|c|c|}
\hline \multirow[t]{2}{*}{ Case } & \multirow[t]{2}{*}{$M / M_{\odot}$} & \multirow[t]{2}{*}{ Type } & \multicolumn{3}{|c|}{$\Delta R / R$} & \multicolumn{3}{|l|}{$\Delta L / L$} & \multicolumn{3}{|l|}{$\Delta \rho_{\mathrm{c}} / \rho_{\mathrm{c}}$} & \multicolumn{3}{|l|}{$\Delta T_{\mathrm{c}} / T_{\mathrm{c}}$} \\
\hline & & & $\overline{\mathrm{A}}$ & B & $\mathrm{C}$ & $\bar{A}$ & B & $\mathrm{C}$ & $\overline{\mathrm{A}}$ & B & $\mathrm{C}$ & $\bar{A}$ & B & $\mathrm{C}$ \\
\hline C1.1 & 0.9 & MS & -0.01 & 0.10 & -0.08 & -0.03 & -0.05 & -0.02 & 0.00 & -0.17 & -0.17 & -0.01 & -0.02 & -0.007 \\
\hline C1.2 & 1.2 & ZAMS & -0.05 & -0.12 & -0.07 & 0.04 & -0.06 & -0.10 & -0.001 & -0.17 & -0.17 & 0.006 & -0.04 & -0.05 \\
\hline $\mathrm{C} 1.3$ & 1.2 & SGB & 0.01 & 0.33 & 0.31 & 0.36 & 0.90 & 0.53 & -0.30 & -2.5 & -2.2 & -0.02 & 0.55 & 0.57 \\
\hline $\mathrm{C} 1.4$ & 2.0 & PMS & -0.16 & -0.13 & 0.04 & 0.26 & 0.20 & 0.04 & -0.16 & 0.47 & -0.30 & 0.00 & 0.00 & 0.00 \\
\hline $\mathrm{C} 1.5$ & 2.0 & TAMS & -0.03 & 0.43 & 0.46 & 0.76 & 0.82 & 0.07 & -0.20 & -0.30 & -0.10 & 0.06 & 0.05 & -0.007 \\
\hline C1.6 & 3.0 & ZAMS & -0.14 & -0.14 & 0.00 & 0.14 & -0.14 & 0.00 & -0.06 & -0.05 & 0.00 & 0.01 & -0.008 & 0.01 \\
\hline $\mathrm{C} 1.7$ & 5.0 & MS & 0.05 & 0.18 & 0.13 & 0.003 & 0.25 & 0.25 & -0.005 & -0.05 & -0.05 & 0.000 & 0.02 & 0.02 \\
\hline
\end{tabular}

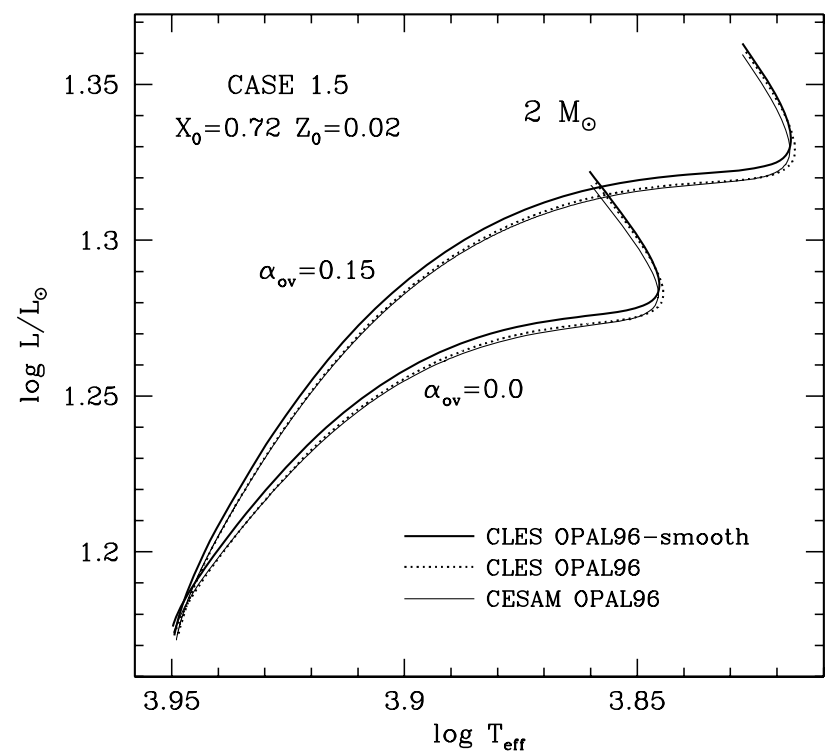

Fig. 6 Evolutionary tracks for stellar parameters corresponding to the case C1.5 in Task 1, and also without overshooting. Solid thick lines: CLÉS models with the default opacity tables (OPAL96-S); solid thin lines: CESAM models; dotted lines: CLÉS models where the opacity tables have been recomputed without using the opal smoothing filter (OPAL96)

$X_{\text {CLES-OPAL96-S }}-X_{\text {CLES-OPAL96, }}$, and columns B and C the differences with CESAM, that is, $X_{\mathrm{CLES}-\mathrm{OPAL} 96-\mathrm{S}}-$ $X_{\text {CESAM }}$ and $X_{\text {CLES-OPAL96 }}-X_{\text {CESAM }}$ respectively.

We note that for the most evolved models (C1.3 and C1.5), the effect on the radius of changing the CLÉS opacity tables is small, and that the agreement with CESAM gets even worse than with the original tables. There is however a significant decrease of the luminosity discrepancy. For the cases C1.4 and C1.6 (PMS and ZAMS respectively) the change from OPAL96-S to OPAL96 is particularly effective, leading to a decrease of $\Delta R$ and $\Delta L$ by a factor 4 and 6 respectively for $\mathrm{C} 1.4$, and dropping the discrepancy to values lower than $0.003 \%$ for C1.6.

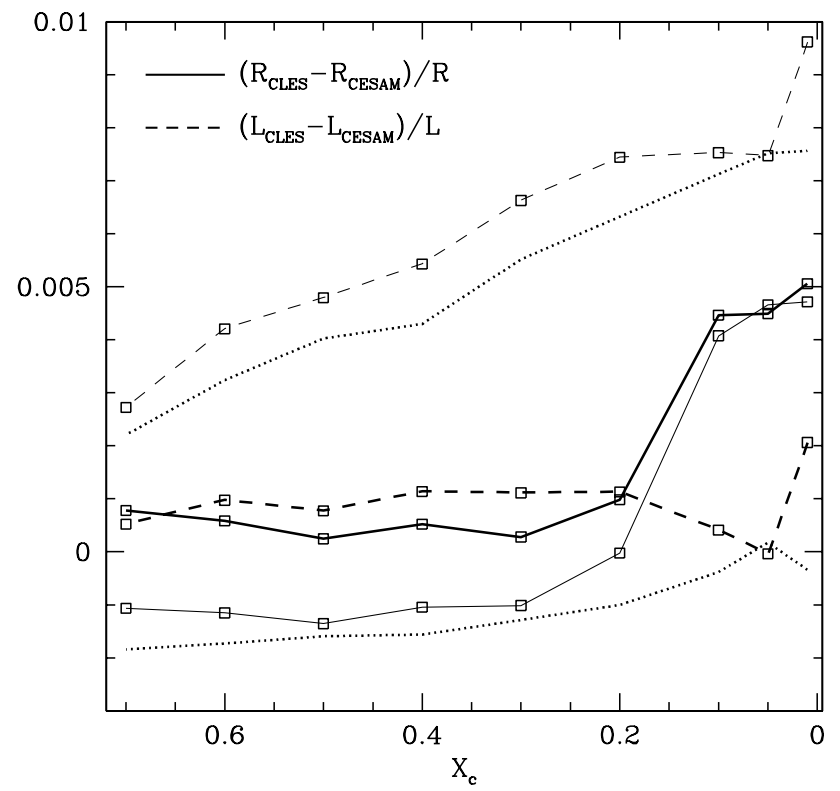

Fig. 7 Effect of opacity uncertainties on the stellar radius and luminosity along the main-sequence evolution of a $2 M_{\odot}$ model. Thin lines correspond to differences between CESAM and CLÉS-OPAL96-S global parameters, and thick ones to differences between CESAM and CLÉS-OPAL96 ones. The dotted lines refer to differences in radius (lower curve) and in luminosity (upper curve) between CLÉS models computed with OPAL96-S and OPAL96 opacity tables

We also studied the effect of opacity tables on the mainsequence evolution of a $2 M_{\odot}$ star (parameters corresponding to C1.5). As shown in Fig. 6 the HR location of the CLÉS-OPAL96-S evolutionary track is significantly modified by adopting OPAL96 tables, and except for the second gravitational contraction, the new track coincides quite well with the CESAM one. The discrepancies in radius and luminosity along the MS as well as the effect of opacity tables on their values are shown in Fig. 7. These discrepancies are significantly reduced by switching from OPAL96-S to OPAL96, nevertheless the difference in the stellar radius at 

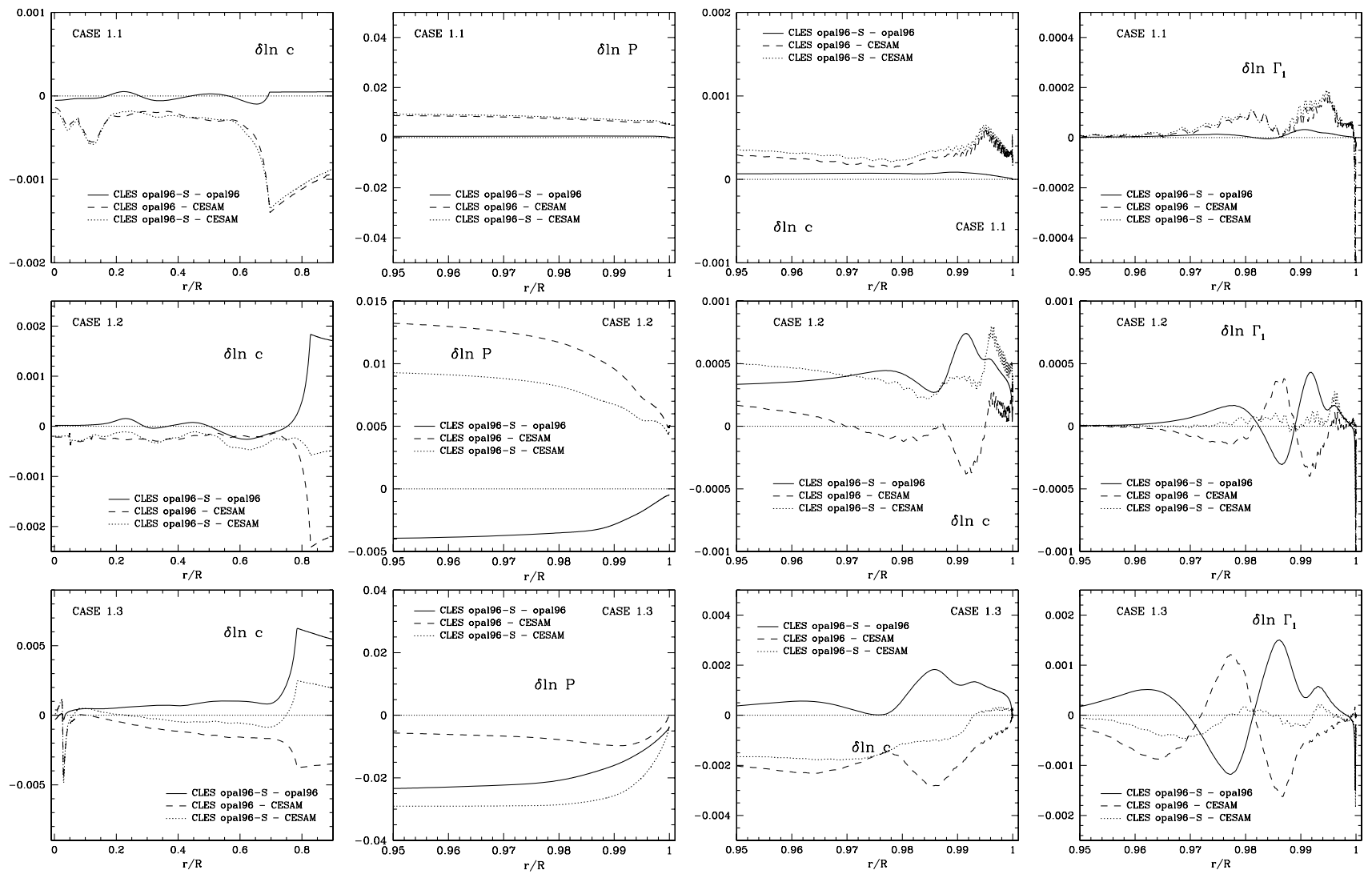

Fig. 8 Plots in terms of the relative radius of the differences at fixed relative mass (two left panels) for the internal regions, and at fixed radius (two right panels) for the outer layers, between models computed with different opacity routines for the Cases 1.1, 1.2 and 1.3. Solid lines correspond to the difference between two types of CLÉS models: those obtained with the standard CLÉS version that uses the smoother OPAL opacity tables (opal96-S) and those obtained by using an OPAL opacity

the end of MS phase $\left(X_{\mathrm{c}}<0.2\right)$ is unchanged. That is not due to the uncertainties in opacity as shown by the dotted lines that correspond to the differences between CLÉS models computed with the two different opacity tables. Neither it is a consequence of the treatment of overshooting since stellar models computed without overshooting for the same stellar parameters show similar discrepancies. The reason is in the treatment of the borders of convective regions in CLÉS that leads to a sort of "numerical diffusion" (Scuflaire et al. 2008a). For the stellar evolution, this diffusion at the border of the convective core works as a slightly larger overshooting. A significant increase of the number of mesh points used for computing the models reduces the numerical diffusion and improves the agreement CLÉS-CESAM.

\subsection{Effects on the stellar structure}

To analyze to which extent the differences reported in (Lebreton et al. 2008a) come from the uncertainty in the opacity,

table obtained without smoothing. Dotted lines: differences between the standard CLÉS models and those from CESAM. Dashed lines: differences between CLÉS models computed by using OPAL opacity tables without smoothing and CESAM models. Left panel: logarithmic sound speed differences. Central left panel: logarithmic pressure differences. Central right panel: logarithmic sound speed differences. Right panel: logarithmic adiabatic exponent differences

we computed for each TASK 1 model the local differences in the physical variables at fixed relative mass and at fixed relative radius. To this purpose we use the so-called difffgong. $d$ routine in the ADIPLS package. ${ }^{1}$ In Figs. 8 and 9 we plot the logarithmic differences of the sound speed, $c$, and pressure, $P$, for the stellar interior (two left panels) and of sound speed and the adiabatic exponent $\Gamma_{1}$ in the external layers. In each panel there are three different curves that correspond to the comparisons labeled $\mathrm{A}, \mathrm{B}$, and $\mathrm{C}$ in previous section. So, if the differences shown in Lebreton et al. (2008a) come from the differences in the opacity tables, the solid and dotted lines should be close to each other. We call again the attention to the improvement got for the cases $\mathrm{C} 1.4$ and C1.6.

\footnotetext{
${ }^{1}$ http://www.corot.pt/ntools.
} 

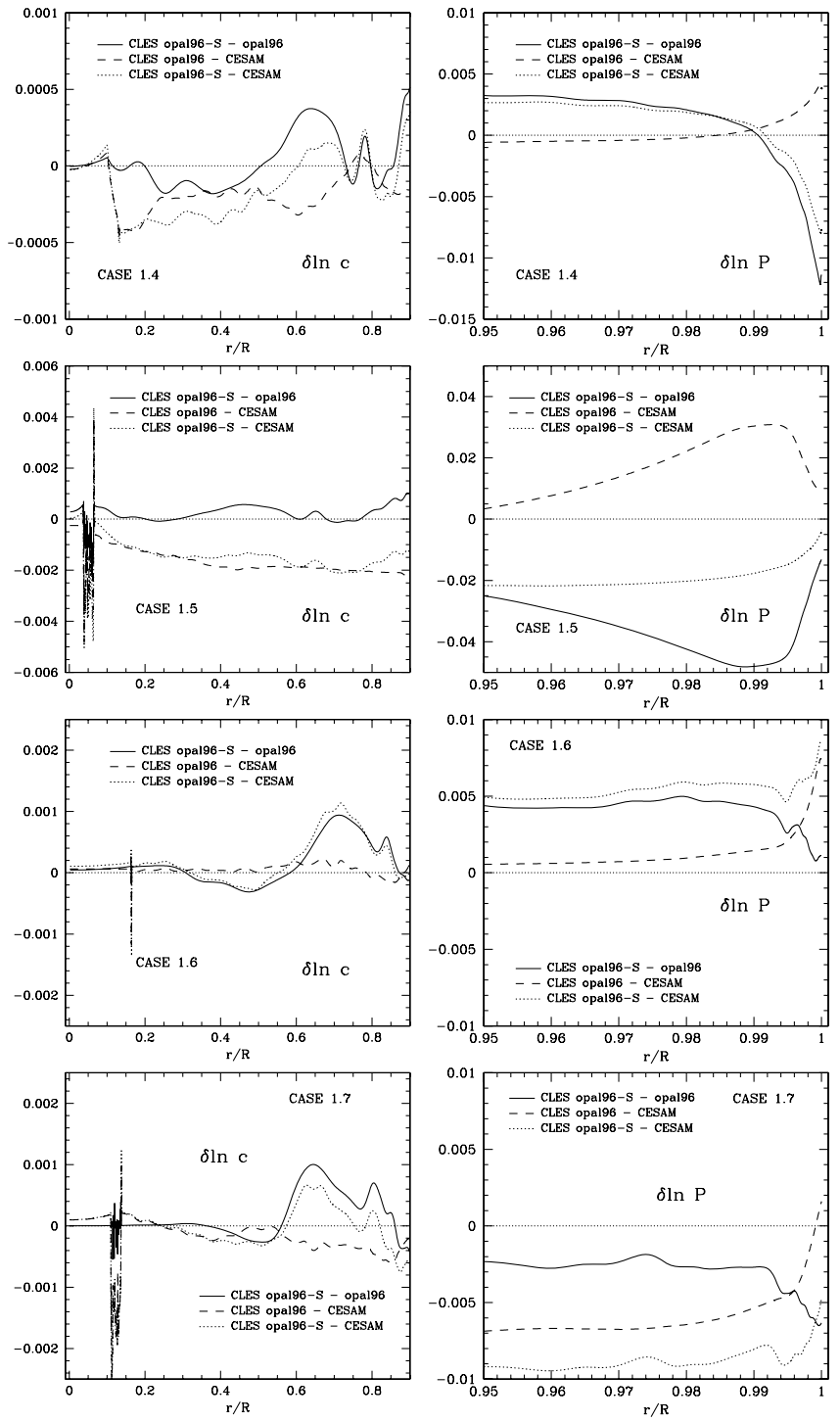
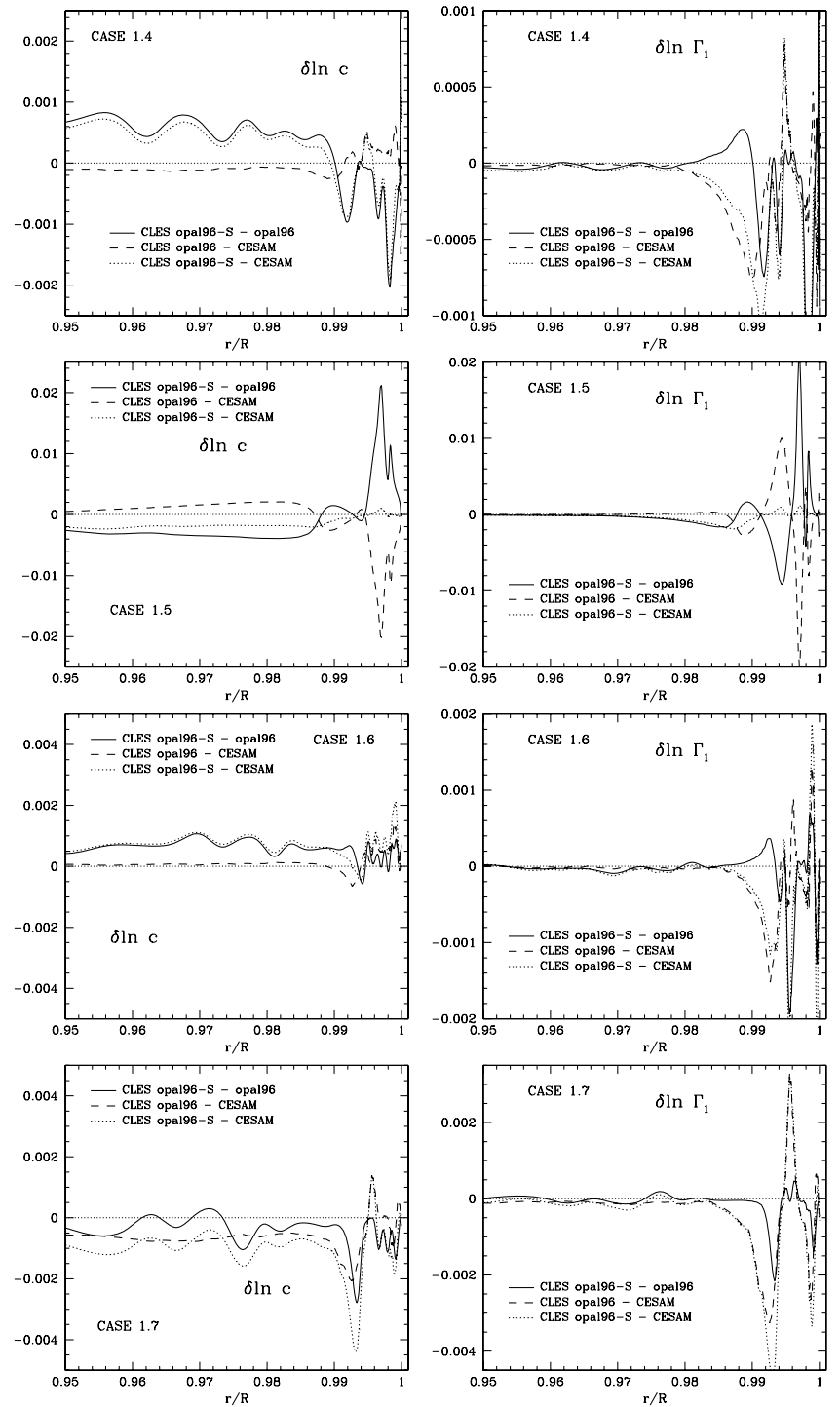

Fig. 9 As Fig. 10, for Cases 1.4, 1.5, 1.6 and 1.7

\subsection{Effects on the frequencies}

Since the frequency of p-modes firstly depends on the stellar radius, the improved agreement between CESAM and CLÉS models that we obtained by changing from OPAL96-S to OPAL96 implies also a decrease in the frequency discrepancies. The values of $\Delta v\left(v_{\text {CLES }}-v_{\text {CESAM }}\right)$ change from 7.25 to $6.35 \mu \mathrm{Hz}$ (at $5300 \mu \mathrm{Hz}$ ) for the case C1.1; from 7 to $4.5 \mu \mathrm{Hz}$ (at $4000 \mu \mathrm{Hz}$ ) for $\mathrm{C} 1.2$; from -5 to $-4.5 \mu \mathrm{Hz}$ at $1000 \mu \mathrm{Hz}$ (and from -11 to -10 at $2300 \mu \mathrm{Hz}$ ) for $\mathrm{C} 1.3$; from 3.7 to $-1 \mu \mathrm{Hz}$ at $2200 \mu \mathrm{Hz}$ for $\mathrm{C} 1.4$. The improvement is only of $0.3 \mu \mathrm{Hz}$ for the case $\mathrm{C} 1.5$, and $\Delta v$ at $1200 \mu \mathrm{Hz}$ is of the order of $7.5 \mu \mathrm{Hz}$. For C1.6 the initial $\Delta v \sim 3 \mu \mathrm{Hz}$ at $1500 \mu \mathrm{Hz}$ drops to values lower than $0.05 \mu \mathrm{Hz}$, and for C1.7, $\Delta v$ change from $1.6 \mu \mathrm{Hz}$ to $1.2 \mu \mathrm{Hz}$ (at $600 \mu \mathrm{Hz}$ ).
By comparing frequencies that have been scaled to the same radius we remove the effect of $\Delta R$ and make appear the differences due to discrepancies in the stellar structure. This was done in Fig. 10 where we plot the frequency differences for $\ell=0$ and 1 and for the cases considered in Task 1 . There are two curves in each panel, one corresponding to the difference $v_{\text {CLES-OPAL96-S }}-v_{\text {CESAM }}$ (that is, that appearing also in Lebreton et al. 2008a), and the second one corresponding to $v_{\text {CLES-OPAL96 }}-v_{\text {CESAM }}$.

The role of opacity on the oscillation frequencies is a intricate problem since the variations of $\kappa$ lead to changes of the temperature structure in the star, and therefore also of the value of $\Gamma_{1}$. As can be seen in Fig. 9 for the case 1.5, the differences in the outer layers might even increase for the model computed with similar opacity tables (OPAL96), and as consequence, the frequency differences scaled to the 

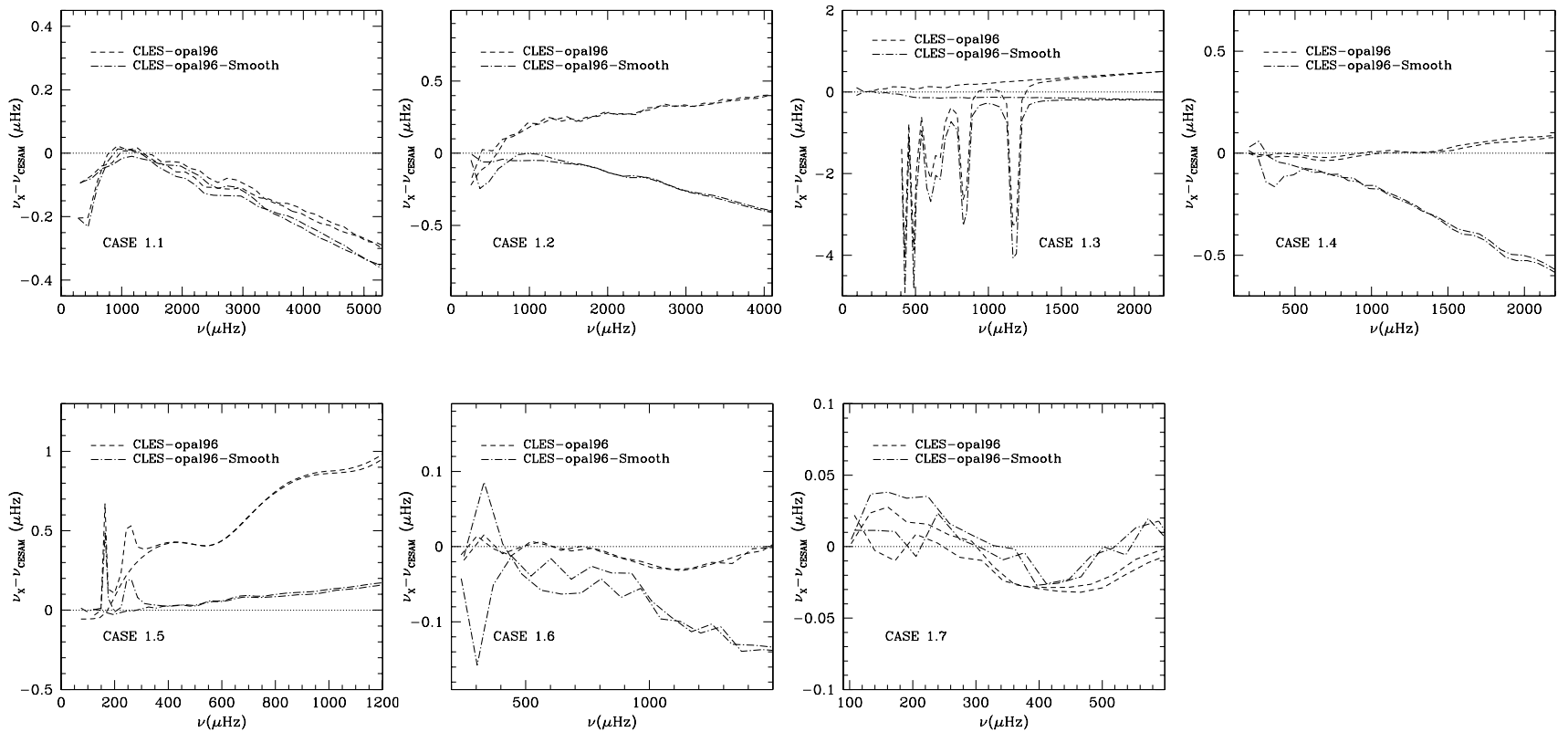

Fig. 10 p-mode frequency differences between TASK 1 models produced by CLES with the two kinds of opacity tables (dash-dotted lines: smoothed OPAL; and dashed lines: without smoothing) and CESAM.

same radius (Fig. 10, lower-left panel) show a discrepancy even larger than with OPAL96-S. The oscillatory behavior is produced by the peak in $\delta \ln c$ at $r / R \sim 0.997$. A comparison between CLÉS-OPAL96 and CLÉS-OPAL96-S clearly shows the same oscillatory behavior, but the absolute frequency difference is only $\sim 0.3 \mu \mathrm{Hz}$ (both models have similar radius). In the same way, CESAM and CLÉS $2 M_{\odot}$ models at $X_{\mathrm{c}}=0.50$ (whose radius differ by less than $3 \times 10^{-4} R_{*}$ once CLÉS adopts OPAL96 tables) show frequency differences of the order of $0.4 \mu \mathrm{Hz}$ at $1200 \mu \mathrm{Hz}$. After normalizing to the same radius an oscillatory component (amplitude $0.01 \mu \mathrm{Hz}$ ) remains in the $\Delta v$ because of the differences in $\Gamma_{1}$ in the outer layers.

\section{Nuclear reaction rates}

We used the basic pp and $\mathrm{CNO}$ reaction networks up to the ${ }^{17} \mathrm{O}(\mathrm{p}, \alpha){ }^{14} \mathrm{~N}$ reaction. In the present models the CESAM code takes ${ }^{7} \mathrm{Li},{ }^{7} \mathrm{Be}$ and ${ }^{2} \mathrm{H}$ at equilibrium while CLÉS follows entirely the combustion of ${ }^{7} \mathrm{Li}$ and ${ }^{2} \mathrm{H}$. The nuclear reaction rates are computed using the analytical formulae provided by the NACRE compilation (Angulo et al. 1999). CESAM uses a pre-computed table ${ }^{2}$ while CLÉS uses directly the analytical expressions. Comparing the nuclear reaction rates for a $2 M_{\odot}$ stellar structure and a given chemical composition, we found that the relative differences are

\footnotetext{
${ }^{2}$ By using pre-computed tables the numerical coherence of derivatives required in the CESAM numerical scheme are guaranteed.
}

The latter is taken as reference, and the frequencies have been scaled to remove the effect of different stellar radii. For each model we plot two curves corresponding to modes with degrees $\ell=0$ and $\ell=1$

of the order of $3 \times 10^{-4}-2 \times 10^{-3}$ (except for nuclear reactions involving ${ }^{7} \mathrm{Li}$ ) if screening factors are included, and of the order or $10^{-8}$ if not. In both codes weak screening is assumed under Salpeter (1954)'s formulation. The screening factor is written $f=\exp \left(A z_{1} z_{2} \sqrt{\frac{\rho \xi}{T^{3}}}\right)$ where $z_{1}$ and $z_{2}$ are the charges of the interacting nuclei. CESAM uses the expression 4-221 of Clayton (1968) where $A=1.88 \times 10^{8}$, $\xi=\sum_{i} z_{i}\left(1+z_{i}\right) x_{i}$, and $x_{i}$ is the abundance per mole of element $i$. The standard version of CLÉS code takes $A=1.879 \times 10^{8}$ and $\xi=\sum_{i=1}^{4} z_{i}\left(1+z_{i}\right) x_{i}+Z(1+Z) x(Z)$ where $x(Z)$ is the abundance of an "average" element containing all the elements different from hydrogen and helium, and $Z$ is the average charge of this element. This approximate estimation of $\xi$ has been changed in CLÉS by assuming full ionization and taking the contribution from each mixture element into account. With this new prescription the differences between CESAM and CLÉS nuclear reaction rates are still of the same order, but with CESAM values larger than CLÉS ones at variance with what was found with the standard CLÉS formulation.

All the Task 1 and Task 3 CLÉS-models were computed with the updated version.

\section{Atmosphere}

Eddington's grey $T(\tau)$ law is used for the atmosphere calculation: $T=T_{\text {eff }}\left[\frac{3}{4}\left(\tau+\frac{2}{3}\right)\right]^{\frac{1}{4}}$ where $\tau$ is the optical depth. CESAM integrates the hydrostatic equation in the atmosphere 


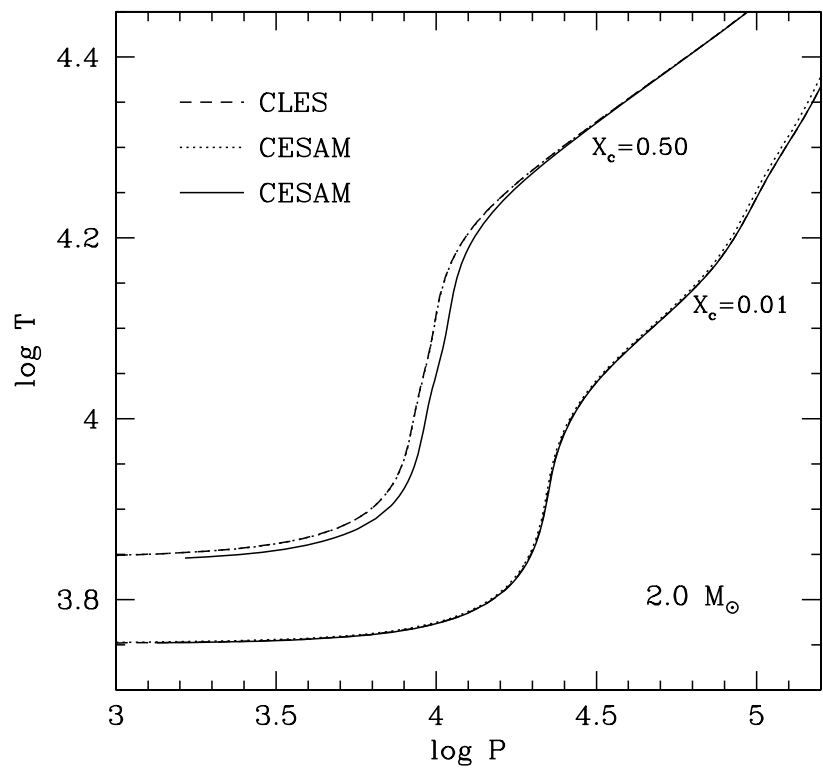

Fig. 11 Stellar structure of the external layers of $2 M_{\odot}$ models at two different evolutionary stages $\left(X_{c}=0.50\right.$ and 0.01$)$. Dashed lines: CLÉS models. Dotted lines: CESAM models with boundary conditions given by $P\left(\tau_{\min }\right)$. Solid lines: CESAM models with boundary conditions given by $\rho\left(\tau_{\min }\right)$ and default values (see the text)

starting at the optical depth $\tau=\tau_{\min }\left(\tau_{\min }=10^{-4}\right.$ for solar like models) and makes the connection with the envelope at $\tau=10$ where the continuity of the variables and of their first derivatives are assured. The radius of the star is taken to be the bolometric radius, i.e. the radius at the level where the local temperature equals the effective temperature $(\tau=2 / 3$ for the Eddington's law).

In the stellar structure integration CLÉS gets the external boundary conditions (the values of density and temperature at a given optical depth $\tau$ ) by interpolating a pre-computed table, and the stellar radius is defined as the level where $T=$ $T_{\text {eff }}$. The Eddington atmosphere table, which provides $\rho$ and $T$ at $\tau=2 / 3$ (therefore at $R=R_{*}$ ), was built by integrating the hydrostatic equilibrium equation in the atmosphere starting at an optical depth that can vary between $10^{-4}$ and $10^{-2}$. The atmosphere structure for a given model is computed afterwards, by integrating the same equations for the corresponding values of $T_{\text {eff }}, \log g$ and chemical composition.

While in CLÉS atmosphere computations the condition at the optically thin limit $\left(\rho\left(\tau_{\min }\right)\right)$ is determined for each $\left(T_{\text {eff }}, \log g, X, Z\right)$ by a Newton-Raphson iteration algorithm, CESAM allows to integrate the atmosphere by fixing either $\rho\left(\tau_{\min }\right)$ or $P\left(\tau_{\min }\right)$. We think it is worth warning here about the relevance of an appropriate choice of $\rho\left(\tau_{\min }\right)$ in CESAM calculations. As explicitly indicated in the corresponding tutorial, the default values were determined for solar like models, and if much different physical conditions are considered, the boundary conditions in the optically thin limit should be coherently changed.
These comparisons have allowed to show the discrepancies in frequencies that a "black-box" use of an evolution code might lead to. For illustration, in Fig. 11 we show the outer layers of $2 M_{\odot}$ models at two different evolutionary states $\left(X_{\mathrm{c}}=0.50\right.$, and $\left.X_{\mathrm{c}}=0.01\right)$, with effective temperature $T_{\text {eff }}=8337 \mathrm{~K}$ and $6706 \mathrm{~K}$ respectively. While the atmosphere and sub-phostospheric structure of CLÉS and CESAM models is quite close if $P\left(\tau_{\min }\right)$ option is used in CESAM, discrepancies that increase with the effective temperature of the model appear when $\rho\left(\tau_{\min }\right)$ with default values (derived for solar-like models) is adopted. The differences induced in the structure of these outer layers by the use of inappropriate limit values in the atmosphere integration are much larger than those due to opacity differences at $\log T \sim 4$. These outer structure differences can lead in fact to frequency differences of the order of several $\mu \mathrm{Hz}$.

\section{Numerical aspects}

The different numerical techniques in CLÉS and CESAM lead to different distribution of mesh points in the stellar structure and to different values of the time step between two consecutive models. As pointed out in Sect. 3.1 (see also Lebreton et al. 2008a) the disagreement between CLÉS and CESAM models can be partially reduced in some cases by changing the mesh. Even if both sets of models have a similar total number of mesh points, their distribution inside the star, as shown in Fig. 12 (right column), is quite different. In this section we analyze the effect of doubling the number of mesh points (CLÉS-X2) or the number of time steps (CLÉS-T2) on the differences between CLÉS and CESAM. As shown in Fig. 12 the effects of these changes are not the same in all the considered stellar cases. While for the CASE 1.1 the increase of mesh points makes almost disappear the disagreement between CLÉS and CESAM models $\left(\delta \ln\right.$ Var $\left.\sim 10^{-4}-5 \times 10^{-5}\right)$, the effect is almost negligible for the CASE 1.3. Doubling the number of mesh points in CASE 1.5 leads to a significant effect in decreasing the luminosity of this TAMS model. As pointed out in Sect. 3.1, a larger number of mesh points near the boundary of the convective core decreases the effect of the sort of "numerical diffusion" that changes the chemical composition gradient at the boundary of the convective core, and that works as a slightly larger overshooting. In fact, the differences of hydrogen abundance in the region of chemical composition gradient $(r / R$ between 0.035 and 0.06 , Fig. 12 central-left panel for CASE 1.5) also decrease with respect to those obtained with the standard CLÉS models. As already discussed in Sect. 3.1 a part of the disagreement between CLÉS and CESAM comes from the differences in the opacities used in both codes. In the lower panels of Fig. 12 we have also plotted (dotted lines) the results of comparing the models computed with CLÉS doubling the number of mesh points and 

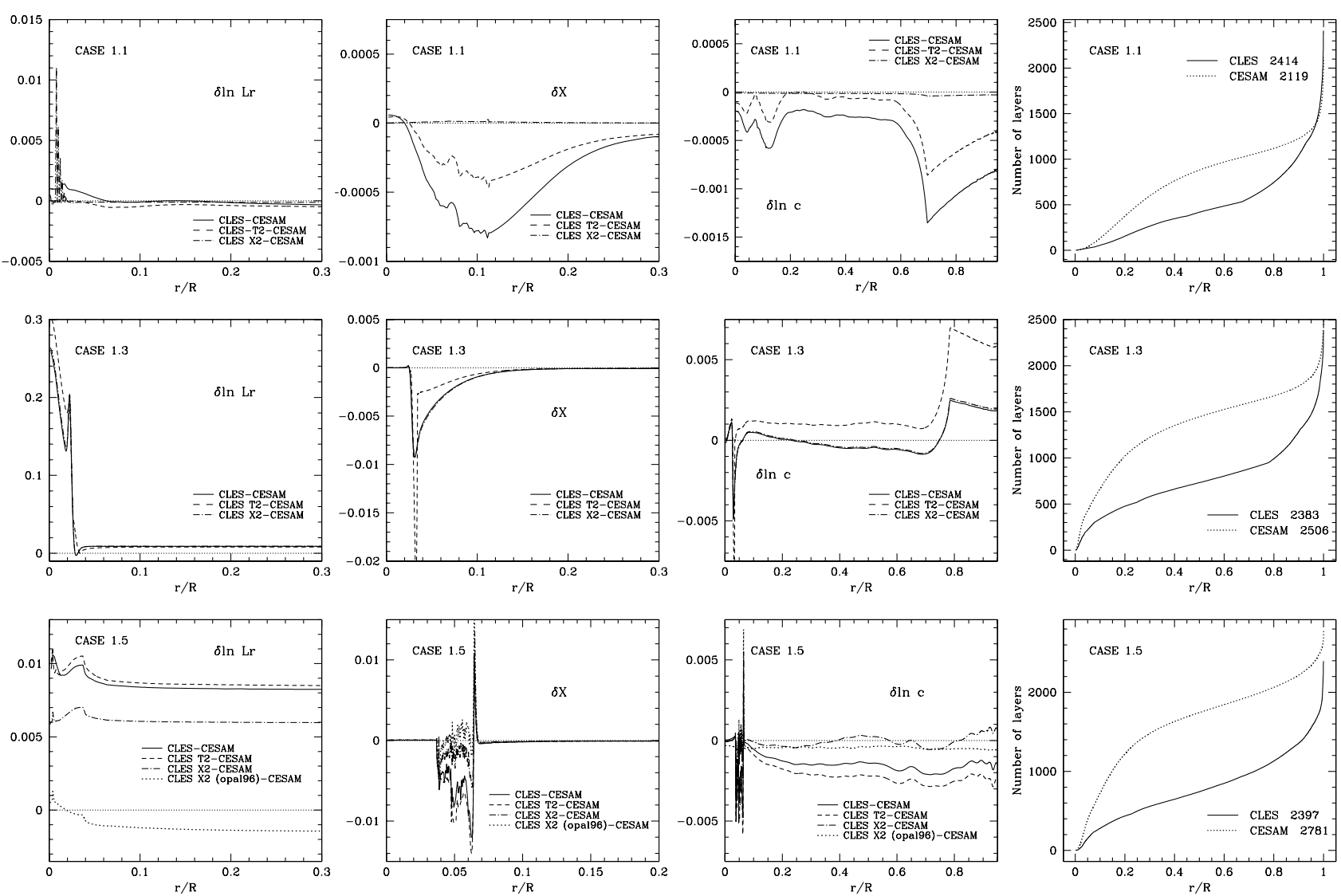

Fig. 12 Plots in terms of the relative radius of the differences at fixed relative mass (three left panels) between three different CLÉS computations and CESAM models. Solid lines correspond to differences between standard CLÉS and CESAM. Dashed lines correspond to differences between CLÉS models computed by doubling the number of time steps and CESAM ones. Dot-dashed lines correspond to differ-

using the OPAL96 tables without smoothing. A significant decrease of luminosity and hydrogen-profiles differences is obtained when both the number of mesh points and the opacity tables are changed.

Decreasing the time step in the evolution models does not lead, in general, to better agreement between CLÉS and CESAM.

\section{Conclusions}

In addition to the quantitative results of code comparison presented in Lebreton et al. (2008a), the analysis of stellar models computed with the codes CESAM and CLÉS has allowed us to reveal some interesting aspects about the two codes, as well as about the input physics, that only a thorough analysis might bring to light. Some of these evidences have led to changes or correction of bugs in the codes, other

ences between CLÉS models computed with a double number of mesh points. In lower panels there the differences between CESAM and CLÉSX2 models computed using the opacity tables without smoothing (opa196) are plotted by using dotted lines. The right column plots show the distribution of mesh points inside the CESAM models (dotted lines) and CLÉS ones (solid lines)

simply allowed us to understand the origin of some differences that were reported in the above mentioned paper.

- The inconsistencies among the thermodynamic quantities in OPAL2001 equation of state tables lead to differences in the stellar models and in the oscillation frequencies larger than the uncertainties due to different interpolation tools. Even if the quantity $C_{V}$ as tabulated in OPAL2001 tables is not used, the remaining inconsistencies among the three adiabatic indices lead to differences between the model computed with a code that takes the thermodynamic quantities directly from OPAL tables (such as CESAM) and a model computed with a code whose thermodynamic variables are derived by the thermodynamic relations and a minimum of tabulated quantities (such as CLÉS). Furthermore, the discrepancies will depend on the choice of tabulated quantities.

- The precision of the theoretical oscillation frequencies is seriously limited by the uncertainties in the opacity computations. 
- Different approaches used to estimate the electron density in CESAM and CLÉS lead to differences in the screening factors that have no relevant effects on the stellar models.

- Even with a simple physics, such as the Eddington's law for gray atmosphere, the details of numerical tools can have significant consequences on the seismic properties of the models.

- The different distribution of mesh points in the models can explain part of the disagreement between CESAM and CLÉS models. An increase of mesh points in the internal regions seems to be required in CLÉS to decrease the differences with CESAM.

Apart from the discrepancies in the screening factors which does not significantly affect the oscillation frequencies, the other factors analyzed here can affect the absolute oscillation frequencies by up to several $\mu \mathrm{Hz}$.

Acknowledgements J.M., A.M., R.S., and A.N. acknowledge financial support from the Belgium Science Policy Office (BELSPO) in the frame of the ESA PREODEX8 program (contract C90199) and from the Fonds National de la Recherche Scientifique (FNRS). P.M. thanks J.P. Marques (Coimbra University) and L. Piau (Brussels University) for their contribution to the OPAL-EoS implementation in CESAM.

\section{References}

Alexander, D.R., Ferguson, J.W.: Low-temperature Rosseland opacities. Astrophys. J. 437, 879-891 (1994). doi:10.1086/175039

Anders, E., Grevesse, N.: Abundances of the elements-meteoritic and solar. Geochim. Cosmochim. Acta 53, 197-214 (1989). doi:10.1016/0016-7037(89)90286-X

Angulo, C., Arnould, M., Rayet, M., Descouvemont, P., Baye, D., Leclercq-Willain, C., Coc, A., Barhoumi, S., Aguer, P., Rolfs, C., Kunz, R., Hammer, J.W., Mayer, A., Paradellis, T., Kossionides, S., Chronidou, C., Spyrou, K., Degl'Innocenti, S., Fiorentini, G., Ricci, B., Zavatarelli, S., Providencia, C., Wolters, H., Soares, J., Grama, C., Rahighi, J., Shotter, A., Lamehi Rachti, M.: A compilation of charged-particle induced thermonuclear reaction rates. Nucl. Phys. A 656, 3-183 (1999)
Boothroyd, A.I., Sackmann, I.J.: Our Sun, IV: the standard model and helioseismology: consequences of uncertainties in input physics and in observed solar parameters. Astrophys. J. 583, 1004-1023 (2003). doi:10.1086/345407

Clayton, D.D.: Principles of Stellar Evolution and Nucleosynthesis. McGraw-Hill, New York (1968)

Grevesse, N., Noels, A.: Cosmic abundances of the elements. In: Origin and Evolution of the Elements (1993)

Iglesias, C.A., Rogers, F.J.: Updated Opal opacities. Astrophys. J. 464, 943 (1996)

Lebreton, Y., Montalbán, J., Christensen-Dalsgaard, J., Roxburgh, I., Weiss, A.: CoRoT/ESTA-TASK 1 and TASK 3 comparison of the internal structure and seismic properties of representative stellar models. Astrophys. Space Sci. (2008a). doi:10.1007/ s10509-008-9740-8

Lebreton, Y., Monteiro, M., Montalbán, J., Moya, A., Baglin, A., Christensen-Dalsgaard, J., M.-J., G., Michel, E., Provost, J., Roxburgh, I., Scuflaire, R., the ESTA Team: The CoRoT evolution and seismic tool activity. Astrophys. Space Sci. (2008b). doi:10.1007/s10509-008-9771-1

Monteiro, M.J.P.F.G., Lebreton, Y., Montalbán, J., Christensen-Dalsgaard, J., Castro, M., Degl'Innocenti, S., Moya, A., Roxburgh, I.W., Scuflaire, R., Baglin, A., Cunha, M.S., Eggenberger, P., Fernandes, J., Goupil, M.J., Hui-Bon-Hoa, A., Marconi, M., Marques, J.P., Michel, E., Miglio, A., Morel, P., Pichon, B., Prada Moroni, P.G., Provost, J., Ruoppo, A., Suarez, J.C., Suran, M., Teixeira, T.C.: Report on the CoRoT evolution and seismic tools activity. In: Fridlund, M., Baglin, A., Lochard, J., Conroy, L. (eds.) The CoRoT Mission. ESA Publications Division, ESA Spec. Publ. 1306, p. 363, December 2006

Rogers, F.J., Nayfonov, A.: Updated and expanded OPAL equation-ofstate tables: implications for helioseismology. Astrophys. J. 576, 1064-1074 (2002). doi:10.1086/341894

Rogers, F.J., Swenson, F.J., Iglesias, C.A.: OPAL equation-of-state tables for astrophysical applications. Astrophys. J. 456, 902-908 (1996). doi:10.1086/176705

Salpeter, E.E.: Electrons screening and thermonuclear reactions. Aust. J. Phys. 7, 373 (1954)

Scuflaire, R., Montalbán, J., Théado, S., Bourge, P.O., Miglio, A., Godart, M., Thoul, A., Noels, A.: The Liège oscillation code. Astrophys. Space Sci. (2008a). doi:10.1007/s10509-007-9577-6

Scuflaire, R., Théado, S., Montalbán, J., Miglio, A., Bourge, P.O., Godart, M., Thoul, A., Noels, A.: CLÉS, Code Liégeois d'Évolution Stellaire. Astrophys. Space Sci. (2008b). doi:10.1007/s10509007-9650-1 